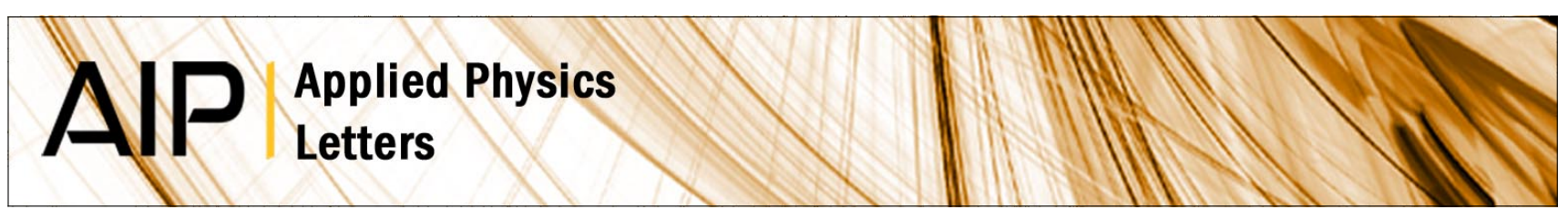

\title{
Synthesis parameter space of bismuth catalyzed germanium nanowires
}

Ying Xiang, Linyou Cao, Jordi Arbiol, Mark L. Brongersma, and Anna Fontcuberta i Morral

Citation: Appl. Phys. Lett. 94, 163101 (2009); doi: 10.1063/1.3116625

View online: http://dx.doi.org/10.1063/1.3116625

View Table of Contents: http://apl.aip.org/resource/1/APPLAB/v94/i16

Published by the AIP Publishing LLC.

Additional information on Appl. Phys. Lett.

Journal Homepage: http://apl.aip.org/

Journal Information: http://apl.aip.org/about/about_the_journal

Top downloads: http://apl.aip.org/features/most_downloaded

Information for Authors: http://apl.aip.org/authors

\section{ADVERTISEMENT}
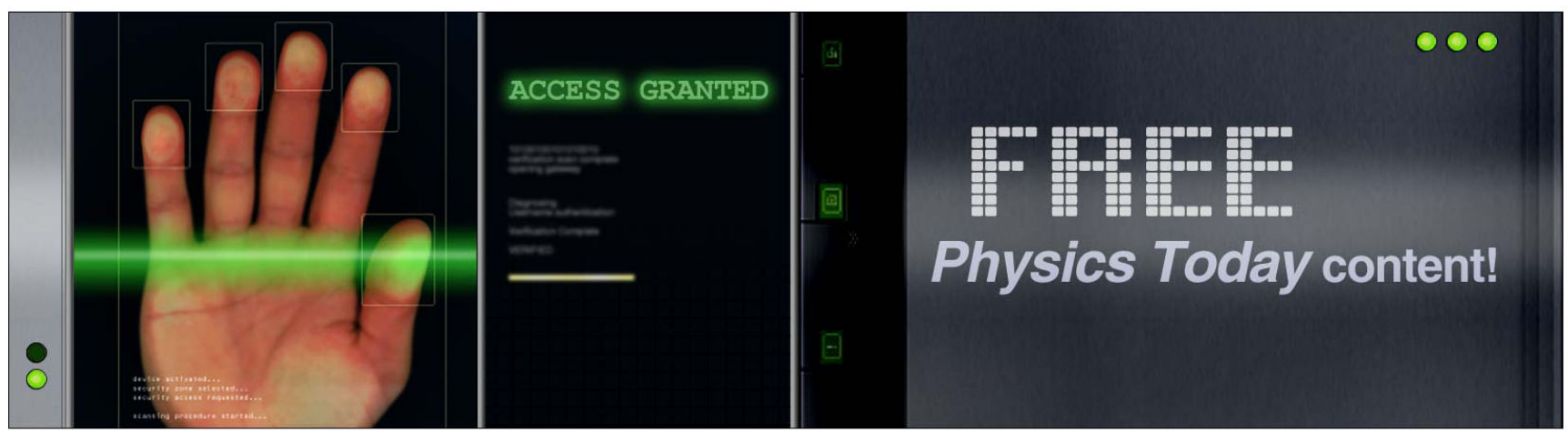


\title{
Synthesis parameter space of bismuth catalyzed germanium nanowires
}

\author{
Ying Xiang, ${ }^{1}$ Linyou Cao, ${ }^{2}$ Jordi Arbiol, ${ }^{3}$ Mark L. Brongersma, ${ }^{2}$ and \\ Anna Fontcuberta i Morral ${ }^{1,4, a)}$ \\ ${ }^{1}$ Walter Schottky Institut, Technische Universität München, Am Coulombwall 3, 85748 Garching, Germany \\ ${ }^{2}$ Geballe Laboratory for Advanced Materials, Stanford University, 476 Lomita Mall, Stanford, \\ California 94305, USA \\ ${ }^{3}$ Department d'electronica, Universitat de Barcelona, 08028 Barcelona, CAT, Spain \\ ${ }^{4}$ Laboratoire des Matériaux Semiconducteurs, Institut des Matériaux, Ecole Polytechnique Fédérale de \\ Lausanne, 1015 Lausanne, Switzerland
}

(Received 6 March 2009; accepted 19 March 2009; published online 20 April 2009)

\begin{abstract}
The synthesis parameter space of bismuth catalyzed germanium nanowires by chemical vapor deposition is determined. The process window for high aspect ratio nanowires is found to be extremely narrow. The optimal conditions are found to be $300{ }^{\circ} \mathrm{C}$ and 150 Torr gas pressure. For lower temperatures, the solubility of $\mathrm{Ge}$ in $\mathrm{Bi}$ is too low for the nucleation of $\mathrm{Ge}$ nanowires to occur. For higher temperatures, small Bi droplets tend to evaporate leading to an extreme reduction in the nanowire density. The extremely low process temperature makes $\mathrm{Bi}$ a good candidate for its growth on low cost and low thermal budget substrates such as plastics. () 2009 American Institute of Physics. [DOI: 10.1063/1.3116625]
\end{abstract}

One-dimensional semiconductors such as quantum wires, nanotubes, and nanowires (NWs) offer exciting possibilities in future high-density electronic circuits, biochemical sensing, nanophotonics, and energy harvesting applications. ${ }^{1-7}$ Of all semiconductor materials, germanium $(\mathrm{Ge})$ is particularly interesting. It possesses a higher carrier mobility than silicon because of the smaller effective mass. ${ }^{8-10}$ Moreover, Ge has a smaller bandgap lying in the infrared at $1.8 \mu \mathrm{m}$, which is desirable for some detector and solar cell applications in which absorption at longer wavelengths is desired. The excitonic Bohr radius of Ge $(24.3 \mathrm{~nm})$ is five times larger than that of $\mathrm{Si}$. As a consequence, quantum confinement effects such as changes in the band structure are easier to observe in Ge than in Si. This, combined with quantum confinement, doping and strain effects that could render it as a direct semiconductor, makes Ge extremely attractive for optical telecommunication applications. ${ }^{11}$

One of the most common synthesis method used for the fabrication of NWs is the vapor-liquid-solid method (VLS), in which a metal catalyst is used to gather and preferentially decompose the growth precursors. Successful growth of NWs has mainly relied on the use of gold $(\mathrm{Au})$ as a catalyst. ${ }^{12-16}$ One important issue for NW synthesis is to find alternative catalysts. Au is a deep level impurity in $\mathrm{Si}$ and $\mathrm{Ge}$ that significantly degrades the carrier mobility and causes fast nonradiative electron-hole pair recombination. ${ }^{5,17,18}$ For this reason it is strongly desired to find alternative metals to $\mathrm{Au}$ that are complementary metal-oxide-semiconductor compatible. ${ }^{18,19}$ From this point of view, $\mathrm{Bi}$ is an interesting candidate because of its low eutectic point $\left(271^{\circ} \mathrm{C}\right)$, shallow doping level, and extremely low solubility in Ge. ${ }^{20}$ Synthesis of Ge NWs employing $\mathrm{Bi}$ as a catalyst has been recently achieved. ${ }^{21}$ The growth was achieved by high temperature sublimation of Ge powder on a transmission electron microscope grid. The processing conditions leading to NW growth have not been explored yet. In order for $\mathrm{Bi}$ to become a

\footnotetext{
${ }^{\text {a) }}$ Author to whom correspondence should be addressed. Electronic mail: anna.fontcuberta-morral@epfl.ch.
}

reliable catalyst for Ge NW growth, it is extremely important that the growth mechanisms and optimized growth conditions are understood by the scientific community. As will be shown, the challenges in using $\mathrm{Bi}$ as a catalyst lie in the facile oxidation of this non-noble metal, extremely low solubility of $\mathrm{Ge}$ and high volatility of $\mathrm{Bi}$.

Ge NWs were synthesized inside a standard thermal chemical vapor deposition furnace using germane $\left(\mathrm{GeH}_{4}\right)$ as a growth precursor. The substrates were fused quartz wafers covered with $1.5 \mathrm{~nm}$ of $\mathrm{Bi}$, which was deposited by electron beam evaporation from a high purity source. Prior to the growth, a $5 \mathrm{~min}$ high temperature anneal was performed at $825^{\circ} \mathrm{C}$, under a flow of 100 SCCM (SCCM denotes standard cubic centimeters per minute at STP) $\mathrm{H}_{2}$ and at a pressure of 30 Torr. $^{22}$ The purpose of the annealing step was to reduce the native $\mathrm{Bi}$ oxide so that the $\mathrm{Bi}$ layer can be exposed to the source gas flow acting as a catalyst for its decomposition. For the Ge NW synthesis, the temperature was lowered and $\mathrm{GeH}_{4}$ (10\% in Ar) was introduced into the growth chamber. The growth parameter space was studied in a temperature range from 270 to $400{ }^{\circ} \mathrm{C}$ and at $\mathrm{GeH}_{4}$ gas pressures between 30 and 300 Torr. No NW growth was observed on bare substrates, eliminating the possibility of catalyst independent NW growth. The NW growth occurrence and morphology was studied by scanning electron microscopy (SEM).

It is generally accepted that the VLS conditions for NW growth can be deduced from the binary phase diagram involving the catalyst and the material grown. The $\mathrm{Bi}-\mathrm{Ge}$

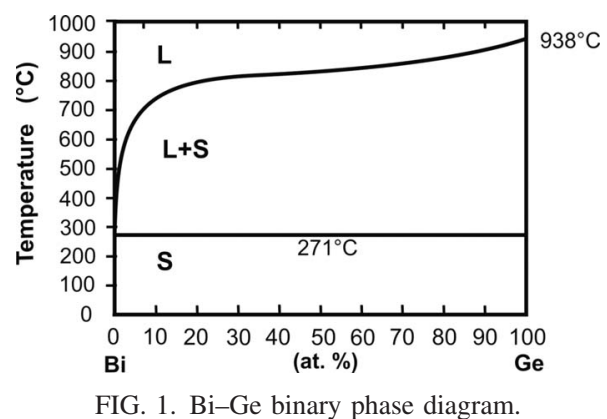



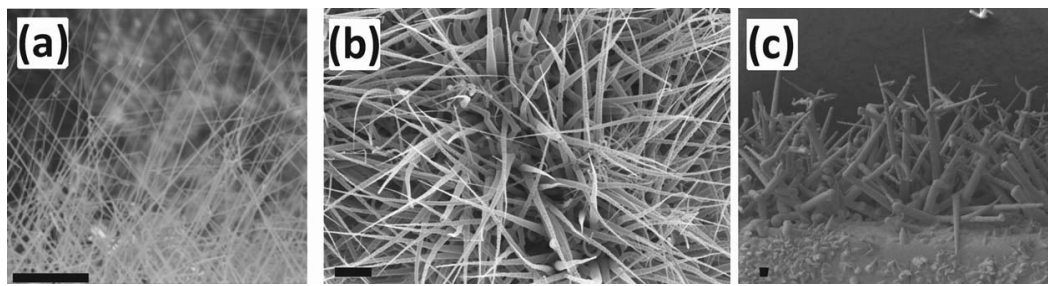

FIG. 2. Scanning electron micrographs of samples grown at 150 Torr and (a) 300, (b) 350, and (c) $400{ }^{\circ} \mathrm{C}$. The scale bar is $10 \mu \mathrm{m}$.

phase diagram is plotted in Fig. 1. It is a single eutectic with the eutectic temperature at $271{ }^{\circ} \mathrm{C}$, which is also the melting temperature of $\mathrm{Bi}$. The eutectic melt is composed of nearly $100 \% \mathrm{Bi}$. The liquidus line defines the composition of a $\mathrm{Bi}-\mathrm{Ge}$ alloy in the liquid state as a function of temperature. For temperatures between 270 and $500{ }^{\circ} \mathrm{C}$, the liquidus line is very close to $100 \% \mathrm{Bi}$ composition, indicating a very poor solubility of Ge. In the nucleation and growth mechanism, the growth precursor diffuses through the droplet to deposit at the solid/liquid interface. ${ }^{23,24}$ For the diffusion to occur, Ge should be soluble in Bi to some extent. The low solubility of Ge implies a difficult nucleation at temperatures close to the eutectic. This differs from what is observed when gold is used as a catalyst. In that case, the solubility of $\mathrm{Ge}$ in the eutectic is 72 at. \% and synthesis below the eutectic temperature is possible. ${ }^{25}$ From the phase diagram alone, it is expected that the NW growth will be the most favorable at higher temperatures, when the solubility of $\mathrm{Ge}$ in $\mathrm{Bi}$ increases.

There are two other factors that affect the Bi-catalyzed growth of Ge NWs: (i) the low vapor pressure of Bi and (ii) the rapid oxidation of $\mathrm{Bi}$ in the presence of oxygen. In the following, we explain how these two factors will affect the growth conditions.

The vapor pressure $P$ of an element determines the evaporation rate as a function of the ambient pressure. It increases dramatically when the curvature of the material is increased, ${ }^{26}$

$$
\Delta P=\frac{2 \gamma M}{r \rho R T} P_{o}(T),
$$

where $\gamma$ is the surface tension, $M$ is the atomic mass, $r$ is the radius, $\rho$ is the density, $R$ is the Arrhenius constant, $T$ is the temperature, and $P_{o}(T)$ is the vapor pressure of a thin film. From this equation, one can deduce that the vapor pressure increases dramatically for droplets of a small radius. The vapor pressure $P_{o}$ of $\mathrm{Bi}$ and its temperature dependence has

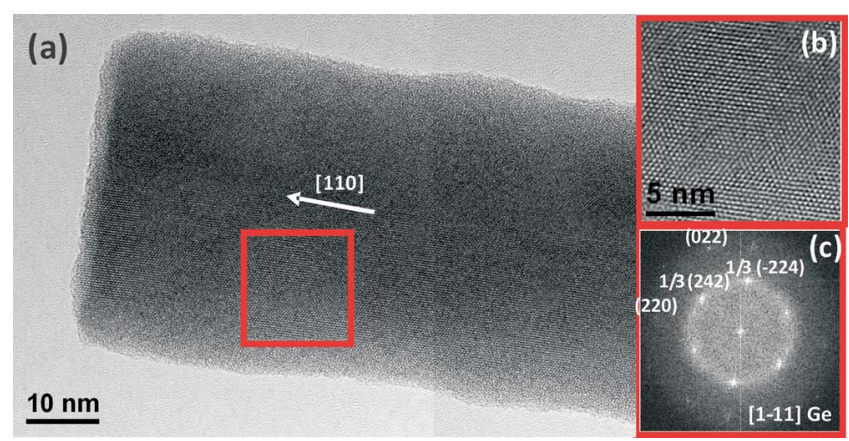

FIG. 3. (Color online) (a) High-resolution transmission electron micrograph of one of the synthesized Bi catalyzed Ge NWs. (b) Magnified detail of the squared region in (a). (c) Indexed power spectra revealing that the NW grows along the [110] axis. been extensively studied in the past. ${ }^{27,28}$ It has been shown that it does not have a simple Arrhenius behavior from 350 to $1000{ }^{\circ} \mathrm{C}$. It is nearly negligible below $350{ }^{\circ} \mathrm{C}$ and increases for higher temperatures, but not monotonically. It has for example a dip around $825{ }^{\circ} \mathrm{C}$. As a consequence, we expect the vaporization of the smallest $\mathrm{Bi}$ droplets for temperatures above $350{ }^{\circ} \mathrm{C}$, resulting in an optimum growth temperature below $350{ }^{\circ} \mathrm{C}$. Moreover, the increase in the vapor pressure may limit the use of higher temperatures for the VLS growth of Ge NWs with $\mathrm{Bi}$ as a catalyst.

Bismuth oxide hinders the catalytic decomposition of $\mathrm{GeH}_{4}$ and therefore also the NW growth. One can limit the oxidation of $\mathrm{Bi}$ or reduce the oxide by exposing it to a reducing ambient such as atomic hydrogen

$$
\mathrm{Bi}_{2} \mathrm{O}_{3}(s)+6 \mathrm{H}(g) \rightarrow 2 \mathrm{Bi}(l)+3 \mathrm{H}_{2} \mathrm{O}(g) .
$$

It has been shown in literature that the decomposition of molecular hydrogen is enhanced at high temperatures and gas pressures. ${ }^{29}$ Consistent with this, we found that catalyst annealing in $\mathrm{H}_{2}$ at high temperatures and a pressure of 30 Torr is a necessary condition to obtain a high yield of NWs on the substrate. In addition, annealing at high temperatures is necessary for the reduction of $\mathrm{Bi}_{2} \mathrm{O}_{3}$, but this treatment should be kept as short as possible in order to avoid the evaporation of the liquid $\mathrm{Bi}$ droplets formed. The temperature of $825^{\circ} \mathrm{C}$ is particularly suitable for this, as there is a dip in the vapor pressure. ${ }^{28}$ It should however be kept as short as possible, in order to avoid the full evaporation of $\mathrm{Bi}$. Without this annealing step, a thin native oxide layer would shield the catalyst, leading to a low yield of NWs. As the synthesis is not realized under ultrahigh vacuum conditions, a small percentage of oxygen is always present in the gas phase. In order to avoid the reoxidation of the Bi droplets, it is important to use conditions in which a certain minimum concentration of atomic hydrogen is ensured.
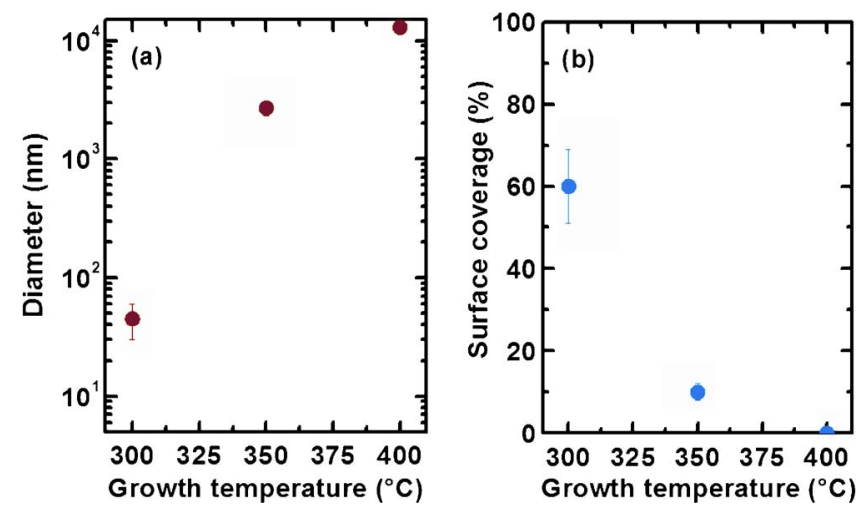

FIG. 4. (Color online) Ge NW diameter and NW density at the surface of the substrate (surface coverage) as a function of growth temperature grown at a $\mathrm{GeH}_{4}$ pressure of 150 Torr. 
The synthesis parameter space of Ge NWs from $\mathrm{Bi}$ as a catalyst was studied. A flow of $1 \mathrm{SCCM} \mathrm{\textrm {H } _ { 2 }}$ and $50 \mathrm{SCCM}$ of $10 \% \mathrm{GeH}_{4}$ in argon were used. The total pressure was varied between 30 and 300 Torr. For all gas pressures, the growth temperatures of $280,300,350$, and $400{ }^{\circ} \mathrm{C}$ were employed. Representative SEM measurements of NW networks obtained at a temperature of 300,350 , and $400{ }^{\circ} \mathrm{C}$ are shown in Fig. 2. At the lowest temperature, no NWs were observed for any of the partial pressures. The failure of NW growth is due to the nearly zero solubility of $\mathrm{Ge}$ in $\mathrm{Bi}$ at temperatures close to the eutectic, as discussed above. From 300 to $400{ }^{\circ} \mathrm{C}$, the NW density tends to increase with the gas pressure. This can result from the higher atomic hydrogen concentration, such as the $\mathrm{GeH}_{4}$ decomposition in $\mathrm{Ge}$ and $\mathrm{H}_{2}$ increases with the gas pressure. The NW diameter and tapering increase dramatically with temperature, as expected from an Arrhenius behavior of chemical reactions. Note that in Fig. 2 the scale bar of each micrograph corresponds to $10 \mu \mathrm{m}$. Highresolution transmission electron microscopy (HRTEM) analysis was performed in order to investigate the NW crystalline structure. Figure 3 shows a typical HRTEM micrograph of a NW synthetized at $300{ }^{\circ} \mathrm{C}$. The NW exhibits the expected diamond cubic structure, with nearly no defects or stacking faults perpendicular to the NW axis. Further analysis by means of the power spectra obtained on the micrographs indicate that the growth direction is the [110] axis. The appearance of $1 / 3\{224\}$ reflections on the power spectra and electron diffraction patterns is usually attributed to the presence of twins and other stacking defects parallel to the observed zone axis (parallel to the NW axis). ${ }^{30}$ The NW diameter and surface density as a function of temperature is shown in Fig. 4. The decrease in NW density and increase in their average diameter could be due to the increase in vapor pressure of $\mathrm{Bi}$ at temperatures higher than $350{ }^{\circ} \mathrm{C}$. This leads to the evaporation of the smaller droplets and prevalence of the bigger ones, helped by a coarsening effect (Ostwald ripening). ${ }^{31}$ This phenomenon is very extreme at temperatures of $400{ }^{\circ} \mathrm{C}$, where the NW growth is only sporadic at the sample surface and the diameter is extremely large (on the order of micrometers). Also, uncatalyzed decomposition rate of $\mathrm{GeH}_{4}$ increases exponentially with temperature. Whenever the rate of Ge deposition onto the catalyst surface is larger than the rate of deposited Ge diffusing away from the catalyst surface, many catalysts stop the growth at certain stages leading to conical NWs, as shown in Fig. 2(c). At temperatures higher than $400{ }^{\circ} \mathrm{C}$ no NW growth was observed. This is due to the complete evaporation of $\mathrm{Bi}$ of the surface. ${ }^{32}$

At last, we discuss the origin of NW tapering for the higher temperatures and higher $\mathrm{GeH}_{4}$ pressures. The tapering of the Ge NWs is characterized by a decreased NW diameter with the distance from the substrate. Aside from the $\mathrm{Bi}$ evaporation, tapering in NWs is due to sidewall deposition of Ge on the NW sidewall. The NW sections near the basis are exposed longer and thus grow thicker. Sidewall deposition is possible when $\mathrm{GeH}_{4}$ is thermally decomposed and its rate increases exponentially with temperature. At higher temperature it turns to be more substantial, explaining that tapering is observed for temperatures higher than $300^{\circ} \mathrm{C}$.

As a conclusion, we have shown that the optimal conditions leading to high density, high aspect ratio Ge NWs are $300{ }^{\circ} \mathrm{C}$ and 150 Torr of gas pressure. At lower temperatures no growth is observed because of the low solubility of Ge in
$\mathrm{Bi}$, while at higher temperatures the NW density decreases and the diameter increase. For temperatures higher than $400{ }^{\circ} \mathrm{C}$ no NW growth is observed, due to the high vapor pressure of $\mathrm{Bi}$.

The authors gratefully acknowledge Gerhard Abstreiter, Ilaria Zardo, and Jason K. Holt for helpful discussions. This work was financially supported by BaCaTec, the Marie Curie Excellence Grant "SENFED," and the Nanosystems Initiative Munich (NIM) of the DFG. The work at Stanford was supported by the Si-based Laser Initiative of the Multidisciplinary University Research Initiative (MURI) under the Air Force Aerospace Research OSR Award No. FA9550-06-10470 and supervised by LTC Gernot Pomrenke.

${ }^{1}$ L. J. Lauhon, M. S. Gudiksen, C. L. Wang, and C. M. Lieber, Nature (London) 420, 57 (2002).

${ }^{2}$ V. Schmidt, H. Riel, S. Senz, S. Karg, W. Riess, and U. Goesele, Small 2, 85 (2006).

${ }^{3}$ L. Zhang, R. Tu, and H. J. Dai, Nano Lett. 6, 2785 (2006).

${ }^{4}$ H. G. Park, C. J. Barrelet, Y. Wu, B. Tian, F. Qian, and C. M. Lieber, Nat. Photonics 2, 622 (2008).

${ }^{5}$ A. R. Guichard, R. D. Kekatpure, M. L. Brongersma, and T. I. Kamins, Phys. Rev. B 78, 235422 (2008).

${ }^{6}$ M. D. Kelzenberg, D. B. Turner-Evans, B. M. Kayes, M. A. Filler, M. C. Putnam, N. S. Lewis, and H. A. Atwater, Nano Lett. 8, 710 (2008).

${ }^{7}$ F. Patolsky, B. P. Timko, G. Zheng, and C. M. Lieber, MRS Bull. 32, 142 (2007).

${ }^{8}$ D. W. Wang, Q. Wang, A. Javey, R. Tu, H. J. Dai, H. Kim, P. C. McIntyre, T. Krishnamohan, and K. C. Saraswat, Appl. Phys. Lett. 83, 2432 (2003).

${ }^{9}$ W. Lu, J. Xiang, B. P. Timko, Y. Wu, and C. M. Lieber, Proc. Natl. Acad. Sci. U.S.A. 102, 10046 (2005).

${ }^{10}$ T. Hanrath and B. A. Korgel, J. Am. Chem. Soc. 126, 15466 (2004).

${ }^{11}$ J. Liu, D. D. Connon, K. Wada, Y. Ishikawa, S. Jonthammanurak, D. T. Danielson, J. Michel, and L. C. Kimerling, Appl. Phys. Lett. 87, 011110 (2005).

${ }^{12}$ R. S. Wagner and W. C. Ellis, Appl. Phys. Lett. 4, 89 (1964).

${ }^{13}$ E. I. Givargizov, J. Cryst. Growth 31, 20 (1975).

${ }^{14}$ D. W. Kwak, H. Y. Cho, and W. C. Yang, Physica E (Amsterdam) 37, 153 (2007).

${ }^{15}$ W. Seifert, M. Borgström, K. Deppert, K. A. Dick, J. Johansson, M. W. Larsson, T. Mårtensson, N. Sköld, C. P. T. Svensson, B. A. Wacaser, L. R. Wallenberg, and L. Samuelson, J. Cryst. Growth 272, 211 (2004).

${ }^{16}$ T. Hanrath and B. A. Korgel, J. Am. Chem. Soc. 124, 1424 (2002).

${ }^{17}$ T. I. Kamins, R. Stanley Williams, Y. Chen, Y. L. Chang, and Y. A. Chang, Appl. Phys. Lett. 76, 562 (2000).

${ }^{18}$ Y. W. Wang, V. Schmidt, S. Senz, and U. Gösele, Nat. Nanotechnol. 1, 186 (2006).

${ }^{19}$ C. Colombo, D. Spirkoska, M. Frimmer, G. Abstreiter, and A. Fontcuberta i Morral, Phys. Rev. B 77, 155326 (2008).

${ }^{20} \mathrm{C}$. Claeys and E. Simoen, Germanium-BasedTechnologies: From Materials to Devices (Elsevier, New York, 2007).

${ }^{21}$ C. Yan and P. S. Lee, J. Phys. Chem. C 113, 2208 (2009).

${ }^{22} 824{ }^{\circ} \mathrm{C}$ is the melting temperature of $\mathrm{Bi}_{2} \mathrm{O}_{3}$.

${ }^{23}$ B. Kalache, P. Roca i Cabarrocas, and A. Fontcuberta i Morral, Jpn. J. Appl. Phys., Part 2 45, L190 (2006).

${ }^{24}$ B. J. Kim, J. Tersoff, S. Kodambaka, M. C. Reuter, E. A. Stach, and F. M. Ross, Science 322, 1070 (2008).

${ }^{25}$ S. Kodambaka, J. Terstoff, M. C. Reuter, and F. M. Ross, Science 316 729 (2007).

${ }^{26}$ C. J. Adkins, An Introduction to Thermal Physics (Cambridge University Press, Cambridge, 1987).

${ }^{27}$ A. T. Aldred and J. N. Pratt, J. Chem. Phys. 38, 1085 (1963).

${ }^{28}$ A. Kawazu, Y. Saito, N. Ogiwara, T. Otsuki, and G. Tominaga, Surf. Sci. 86, 108 (1979).

${ }^{29}$ S. Luidold and H. Antrekowitsch, JOM 59, 54 (2007).

${ }^{30}$ A. H. Carim, K. K. Lew, and J. M. Redwing, Adv. Mater. (Weinheim, Ger.) 13, 1489 (2001).

${ }^{31}$ J. B. Hannon, S. Kodambaka, F. M. Ross, and R. M. Tromp, Nature (London) 440, 69 (2006).

${ }^{32} \mathrm{We}$ have realized annealing studies at the growth temperatures and measured the substrate morphology by atomic force microscopy. For a $30 \mathrm{~min}$ annealing at temperatures $\geq 400 \mathrm{C}$, almost no Bi droplets are found at the surface. 